\title{
The Intraoperative Microlesion Effect Positively Correlates With the Short-Term Clinical Effect of Deep Brain Stimulation in Parkinson's Disease
}

\author{
Stèfan F. Lange, MD $^{1,+}$; Naomi I. Kremer, BS $^{1, \dagger} \oplus$; Teus van Laar, MD, PhD ${ }^{2}$; \\ Fiete Lange, MD, $\mathrm{PhD}^{2}$; T. Elien Steendam-Oldekamp, $\mathrm{MS}^{2}$; \\ D. L. Marinus Oterdoom, MD ${ }^{1}$; Anthony R. Absalom, MBChB, FRCA, MD ${ }^{3}$; \\ J. Marc C. van Dijk, MD, $\mathrm{PhD}^{1}{ }^{\oplus}$; Gea Drost, $\mathrm{MD}, \mathrm{PhD}^{1,2} \odot$
}

\begin{abstract}
Objective: During the surgical procedure of deep brain stimulation (DBS), insertion of an electrode in the subthalamic nucleus (STN) frequently causes a temporary improvement of motor symptoms, known as the microlesion effect (MLE). The objective of this study was to determine the correlation between the intraoperative MLE and the clinical effect of DBS.

Materials and Methods: Thirty Parkinson's disease (PD) patients with Movement Disorder Society (MDS) Unified Parkinson's Disease Rating Scale (UPDRS) part III (MDS-UPDRS III) scores during bilateral STN-DBS implantation were included in this retrospective study. MDS-UPDRS III subscores (resting tremor, rigidity, and bradykinesia) of the contralateral upper extremity were used. During surgery, these subscores were assessed directly before and after insertion of the electrode. Also, these subscores were determined in the outpatient clinic after 11 weeks on average (on-stimulation). All assessments were performed in an offmedication state (at least 12 hours of medication washout).

Results: Postinsertion MDS-UPDRS motor scores decreased significantly compared to preinsertion scores ( $p<0.001$ for both hemispheres). The MLE showed a positive correlation with the clinical effect of DBS in both hemispheres (rho $=0.68$ for the primarily treated hemisphere, $p<0.001$, and rho $=0.59$ for the secondarily treated hemisphere, $p<0.01$ ).

Conclusion: The MLE has a clinically relevant correlation with the effect of DBS in PD patients. These results suggest that the MLE can be relied upon as evidence of a clinically effective DBS electrode placement.

Keywords: Correlation, deep brain stimulation, movement disorders, Parkinson disease, tremor

Conflict of Interest: The authors report no conflict of interest concerning the materials or methods used in this study or the findings specified in this paper.
\end{abstract}

\section{INTRODUCTION}

Deep brain stimulation of the subthalamic nucleus (STN-DBS) is a well-recognized and effective neurosurgical treatment for advanced Parkinson's disease (PD). In patients who are awake during the implantation surgery, the operating team frequently observes an intriguing phenomenon of immediate motor symptom alleviation. It occurs directly after insertion of the electrode in the STN but well before active stimulation. This phenomenon is referred to as the microlesion effect (MLE). Although MLE is a well-known entity, its underlying mechanism remains poorly understood.

It has been suggested that the acute effect of local brain tissue manipulation with the electrode, together with the occurrence of micro-hemorrhages or edema, may contribute to the MLE. ${ }^{1,2}$ Collateral edema, which is associated with inactivation of neurons or fibers surrounding the DBS electrode, and might mimic the effect of a pallidotomy or subthalamotomy, seems to play an important role in the pathophysiology of the MLE. ${ }^{3,4}$ Other, more diffuse, mechanisms could also be responsible for the occurrence of the MLE. One possibility is that leakage of neurotransmitters from damaged synapses might influence the function of the surrounding un-injured neurons. ${ }^{3,5}$ The occurrence of the MLE is hypothesized to be a sign of accurate placement of the DBS

Address correspondence to: Gea Drost, MD, PhD, University Medical Center Groningen, Hanzeplein 1 HPC AB71, 9713 GZ Groningen, The Netherlands. Email: g.drost@umcg.nl

1 Department of Neurosurgery, University of Groningen, University Medical Center Groningen, Groningen, The Netherlands;

2 Department of Neurology, University of Groningen, University Medical Center Groningen, Groningen, The Netherlands; and

${ }^{3}$ Department of Anesthesiology, University of Groningen, University Medical Center Groningen, Groningen, The Netherlands

${ }^{\dagger}$ These authors contributed equally to this study.

For more information on author guidelines, an explanation of our peer review process, and conflict of interest informed consent policies, please see the journal's Guide for Authors.

Source(s) of financial support: This research did not receive any specific grant from funding agencies in the public, commercial, or not-for-profit sectors. 
electrode within the targeted structure. A postoperative MLE is considered to be a good predictor of subsequent DBS treatment effect. $^{1,6-8}$

The current literature has mainly focused on postoperative assessment of the MLE. Observing the patient for an MLE intraoperatively can create the opportunity for timely and possibly more accurate electrode repositioning. The Movement Disorder Society (MDS) revision of the Unified Parkinson Disease Rating Scale (UPDRS) part III (MDS-UPDRS III) is the most frequently used clinical instrument to assess motor function in patients with PD. ${ }^{9}$ The objective of this study was therefore to investigate the correlation between the intraoperatively measured change in MDS-UPDRS III score (the MLE) and the score change measured during follow-up (DBS effect).

\section{MATERIALS AND METHODS}

\section{Study Design}

We reviewed the data of a retrospective cohort of consecutive patients who underwent STN-DBS surgery between April 2014 and November 2020. Motor function was measured at three time points to assess the MLE and the DBS effect: 1) preinsertion and 2) postinsertion of the electrode during surgery, and 3) in the outpatient clinic, on-stimulation (Fig. 1). All assessments were performed in an off-medication state (at least 12 hours of medication washout). According to Dutch legislation, the local research ethics committee granted approval before the study began, and institutional guidelines were followed.

\section{Patients}

Patients eligible for inclusion were adults ( $\geq 18$ years) with advanced PD (persistent motor fluctuations, intractable tremors or medication-induced side effects despite optimal pharmaceutical therapy) who positively responded to levodopa and who underwent awake bilateral STN-DBS surgery using Medtronic 3389 electrodes. Patients had complete preinsertion, postinsertion, and follow-up UPDRS 3.0 or MDS-UPDRS III scores of the same electrode trajectory. Patients were excluded if other neurological or psychiatric conditions were present, if the surgical intervention was a revision of an in situ DBS system, if microrecordings were performed, if more than one electrode insertion attempt per hemisphere was performed, and if concomitant medication was given

Microlesion effect

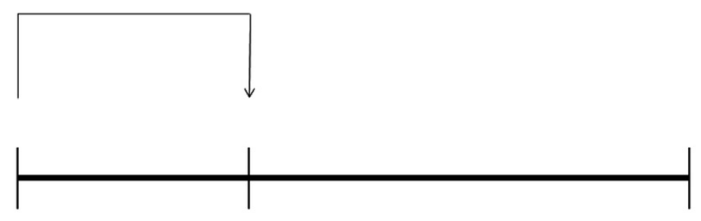

(1) Pre-insertion

(2) Post-insertion

(3) On-stimulation

Figure 1. Study design. Motor function was measured at three time points to assess the MLE and the DBS effect: 1) preinsertion and 2) postinsertion of the electrode during surgery, and 3) in the outpatient clinic, on-stimulation. All assessments were performed in an off-medication state (at least 12 hours of medication washout) during the surgery. All patients were assessed by a panel of neurosurgeons, movement disorder neurologists, and neuropsychologists prior to their STN-DBS surgery.

\section{Surgical Procedure}

Details of the surgical procedure were described previously. ${ }^{10}$ The STN target sites were identified on preoperatively acquired 3 T MRI (Philips Intera, Eindhoven, The Netherlands) images, using the planning software iPlan 3.6 (Brainlab, Feldkirchen, Germany). On the day of the surgery, under standard monitoring, the patient was sedated with a combination of dexmedetomidine and remifentanil. Dexmedetomidine was administered by infusion, starting with a loading dose of $0.5 \mathrm{mcg} / \mathrm{kg}$ administered over $10 \mathrm{~min}$, followed by a maintenance infusion at $0.5 \mathrm{mcg} / \mathrm{kg} / \mathrm{hr}$. Remifentanil was administered by target controlled infusion using an Alaris PK infusion pump (Carefusion/BD, UK) programmed with the Minto model $^{11,12}$ at a target effect-site concentration of $1-2 \mathrm{ng} / \mathrm{mL}$. During the placement of the Leksell G frame (Elekta, Stockholm, Sweden), the pin sites were anesthetized by infiltration of the scalp with a combination of bupivacaine $0.25 \%$ and epinephrine $1: 100,000$. After frame placement, the remifentanil infusion was discontinued, and the dexmedetomidine continued at a rate of $0.3-0.5 \mathrm{mcg} / \mathrm{kg} / \mathrm{hr}$. The patient then underwent $\mathrm{CT}$ imaging (Sensation 64, Siemens, Erlangen, Germany) with the frame in situ. The CT images were transferred to the iPlan software and were then co-registered with the preoperative $3 \mathrm{~T} \mathrm{MRI}$, registering the planned target in the stereotactic coordinate system. The skin incision sites were infiltrated with a mixture of bupivacaine $0.25 \%$ and epinephrine 1:100,000. After incision and prior to drilling of the burrholes, the dexmedetomidine infusion was stopped. Baseline clinical testing was performed followed by implantation of the electrodes. Our standard practice is to first implant the left hemisphere lead. However, in one patient, the right hemisphere lead was implanted first because the left half of the body was more severely affected. Test stimulation was used to assess clinical stimulation effects and stimulation-induced side effects. Microelectrode recording (MER) was not performed. Following bilateral lead placement, CT imaging was repeated to assess lead placement accuracy. Thereafter, general anesthesia was induced and the internal pulse generator was implanted and connected to the leads.

\section{Measurements}

Prior to the surgery, patients were subjected to a levodopa challenge. After overnight withdrawal of dopaminergic medication (at least 12 hours), the UPDRS III was assessed in the off-medication state. After supramaximal dosing of the patient's usual dopaminergic medication (on-medication state), a second assessment was performed. The levodopa response was defined as the difference in total UPDRS III score between the off-medication and on-medication states.

Dopaminergic medication was withheld for at least 12 hours before surgery; thus, patients were in an off-medication state. During surgery, before electrode insertion, baseline motor function was assessed using a modified version of the MDS-UPDRS III. A modified version of the scale was applied, because the patient was on the operating table, and thus, not all items could be performed (eg, gait and postural stability). Resting tremor, rigidity (of the elbow joint), and bradykinesia (finger tapping) were assessed in the contralateral upper extremity (relative to the hemisphere being 
operated) by a movement disorder neurologist. Immediately after insertion of each electrode, this assessment was repeated. Intraoperative scores were noted directly after intraoperative assessment, without knowledge of the follow-up scores.

Follow-up scores were obtained within six months after the surgery, unless the reason for longer follow-up duration was the occurrence of a persisting MLE. In the outpatient clinic, MDS-UPDRS III was assessed in an off-medication/on-stimulation state by a physician assistant trained in performing the MDS-UPDRS. The postoperative rater was blinded to the intraoperative scores.

Total UPDRS 3.0 scores were converted to MDS-UPDRS III scores using the conversion method published by Hentz et al. ${ }^{13}$ UPDRS 3.0 item scores were converted to MDS-UPDRS III item scores using the official conversion formula. ${ }^{9}$

We used compound motor score outcome variables based on the modified version of the MDS-UPRS III. This sum score is the summation of the resting tremor, rigidity, and bradykinesia of the contralateral upper extremity. The MLE was defined as the sum score just prior to electrode insertion minus the sum score immediately post insertion (Fig. 1). The DBS effect was defined as the sum score just prior to electrode insertion minus the sum score at follow-up on-stimulation (Fig. 1). The MDS-UPDRS III scores of the primarily treated hemisphere (the first hemisphere implanted) were considered separately from the MDS-UPDRS III scores of the secondarily treated hemisphere (the second hemisphere implanted), since electrode insertion in the primarily treated hemisphere is known to influence both contra- and ipsilateral parkinsonian symptoms. ${ }^{14,15}$ Therefore, a division between the primarily and secondarily treated hemisphere is preferred above a division between the left and right side.

\section{Statistical Analysis}

Data and statistics are reported separately for the primarily and secondarily treated hemisphere, respectively, unless specified otherwise. Descriptive statistics were used to summarize the data and to identify potential missing data or outliers. All analyses were performed with nonparametric tests, based on visual inspection of the histograms and the low number of observations. Median and range scores were used for descriptive data. A two-sided alpha of 0.05 was used as the level of significance. Statistical analyses were performed using IBM SPSS Statistics version 24.

A Wilcoxon signed-rank test was used to analyze the change in total UPDRS III scores during the levodopa challenge and to analyze the MLE and DBS effects. MLE and DBS effects were defined as stated above (also refer to Fig. 1). The Wilcoxon signed-rank test was also used to analyze differences between the primarily and secondarily treated hemispheres. The correlations between the levodopa response and the MLE with the DBS effect were calculated using Spearman's rho.

\section{RESULTS}

\section{Patients}

Thirty consecutive patients (14 women, 16 men; mean age, $60.2 \pm 7.2$ years) were enrolled in the study (Tables 1 and 2). In one patient, two electrode trajectories were made in the secondarily treated hemisphere. The data from this hemisphere were excluded from analysis, resulting in a total of 59 implanted hemispheres (30 primarily and 29 secondarily treated hemispheres) available for analysis. There was no significant difference between the preinsertion sum scores in the primarily and secondarily treated hemisphere ( 4 vs $4, p=0.60$ ). All but one patient returned to the outpatient clinic within six months. This patient experienced persisting MLE at six months, and therefore his follow-up was postponed until the seventh month. His data were included in the analysis.

\section{The Correlation Between the Levodopa Response and the DBS Effect}

For one patient, the total UPDRS III score was not available to calculate the levodopa response. Therefore, the remaining 29 patients were included in this analysis. Prior to the surgery, the onmedication total UPDRS III score decreased significantly compared to the off-medication total UPDRS III score (38.5 vs $17.0, p<0.001$ ). The levodopa response showed a significant correlation with the DBS effect (rho $=0.44, p=0.02$ ).

\section{Microlesion Effect}

Postinsertion sum scores decreased significantly when compared to preinsertion sum scores (from 4 to 1 vs from 4 to $1.5, p<0.001$, for both hemispheres). The MLE tended to be significantly larger in the primarily treated hemisphere, compared to the secondarily treated hemisphere (3 vs 2, $p=0.054$ ) (Fig. 2, Table 3).

\section{DBS Effect}

The off-medication/on-stimulation sum scores decreased significantly compared to the preinsertion sum scores (from 4 to 1 vs from 4 to $1, p<0.001$, for both hemispheres). There was no significant difference in the DBS effect between the primarily and secondarily treated hemisphere (3 vs $2, p=0.09$ ) (Fig. 2, Table 3).

\section{The Correlation Between the Microlesion Effect and the DBS Effect}

The MLE showed a significant correlation with the DBS effect in both hemispheres ( $r h o=0.68, p<0.001$, and rho $=0.59, p<0.01$ ). Also, when combining both hemispheres, we found a significant correlation between the MLE and the DBS effect (rho $=0.69$, $p<0.001$ ) (Fig. 3).

\begin{tabular}{|c|c|c|}
\hline Demographic & Value & \\
\hline Sex & 14 women & 16 men \\
\hline Mean age (SD) & $60.2(7.2)$ years & \\
\hline Mean disease duration (SD) & 10.4 (3.0) years & \\
\hline $\begin{array}{l}\text { Mean follow-up in } \\
\text { months (SD) }\end{array}$ & $2.6(1.4)$ & \\
\hline Included hemispheres & Left: 30 (100\%) & Right: 29 (97\%) \\
\hline Primarily treated hemisphere & Left: 29 (97\%) & Right: 1 (3\%) \\
\hline Most affected hemisphere & Left: 15 (50\%) & Right: 15 (50\%) \\
\hline $\begin{array}{l}\text { Primarily treated } \\
\text { hemisphere = most } \\
\text { affected hemisphere }\end{array}$ & $16(53 \%)$ & \\
\hline \multirow{5}{*}{ Dominant symptom(s) } & Bradykinesia & $15(50 \%)$ \\
\hline & Rigidity & $4(13 \%)$ \\
\hline & Rest tremor & $4(13 \%)$ \\
\hline & Rigidity and bradykinesia & $5(17 \%)$ \\
\hline & Bradykinesia and tremor & $2(7 \%)$ \\
\hline
\end{tabular}


Table 2. Preinsertion Motor Scores.

\begin{tabular}{|lcl|} 
& $\begin{array}{l}\text { Primarily treated } \\
\text { hemisphere; } \\
\text { median (min-max) }\end{array}$ & $\begin{array}{l}\text { Secondarily treated } \\
\text { hemisphere; } \\
\text { median (min-max) }\end{array}$ \\
Preinsertion sum score & $4(0-8)$ & $4(0-8)$ \\
Preinsertion rigidity & $1.5(0-4)$ & $2(0-2)$ \\
Preinsertion bradykinesia & $2(0-4)$ & $2(0-4)$ \\
Preinsertion rest tremor & $0(0-4)$ & $0(0-3)$ \\
\hline
\end{tabular}

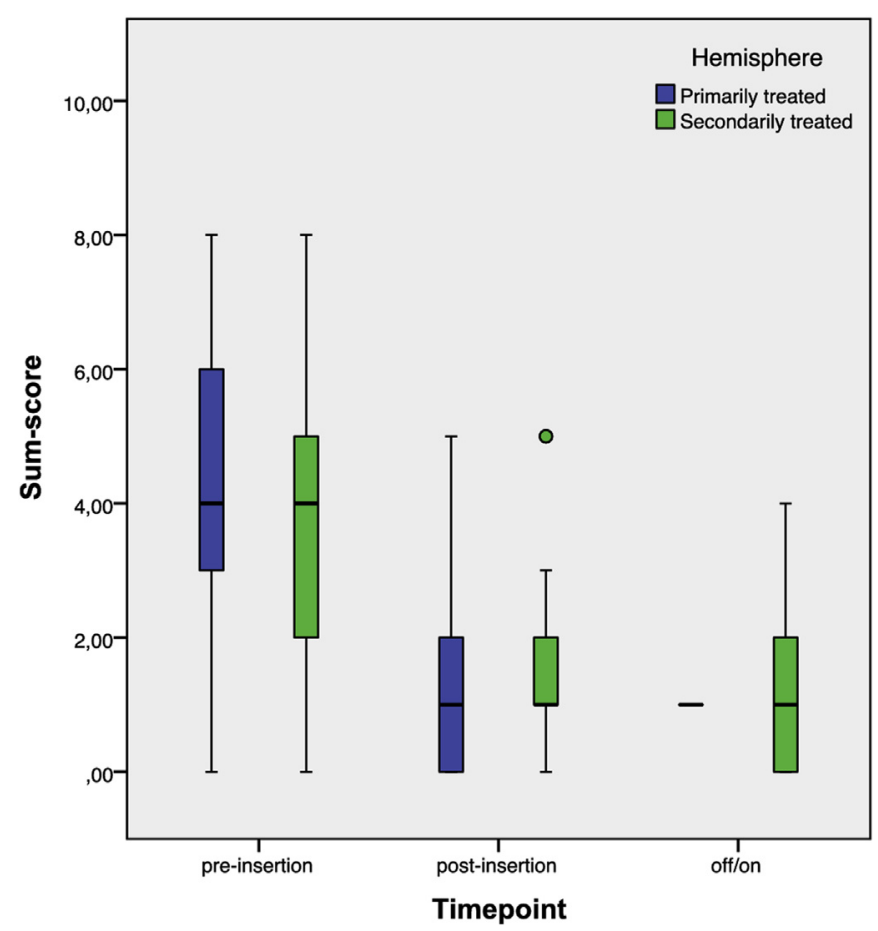

Figure 2. Boxplots with median and interquartile range for sum scores for the primarily and secondarily treated hemisphere. Primarily treated hemisphere during off-medication/on-stimulation: since $57 \%$ of the patients had the sum score 1, the median, first quartile, and third quartile all had the value of 1 (or close to 1), represented by the horizontal line. The following outliers for this group were removed from the graph: subjects 9, 14, 19, 20, 21, and 27 (sum score 0); subjects 12, 26, and 29 (sum score 2); subjects 4 and 28 (sum score 3); subject 6 (sum score 4); and subject 16 (sum score 5). The outliers were not removed from the analysis.

\section{DISCUSSION}

This study confirms the presence of an intraoperatively quantifiable MLE, which correlates with the DBS effect at follow-up in both hemispheres.

In our study, the MLE was assessed intraoperatively, which allowed for direct measurement of the effects of STN penetration by the electrode. To the best of our knowledge, this is the first study evaluating MLE without interference of MER. Assessing both MER and DBS electrode lesion effects could result in bias, since multiple lesion effects are intertwined. All MDS-UPDRS III scores were performed (or supervised in the outpatient clinic) by the same movement disorder neurologist, ruling out inter-rater variability.
Ratings were performed in a blinded setting; the intraoperative scores were not known during follow-up assessment.

The MLE that we observed in this study consisted of reductions in the severity of resting tremor, bradykinesia, and rigidity, consistent with the findings reported previously by Tykocki et al, Maltête et al, and Mann et $\mathrm{al}^{6,7,16}$ In general, the MLE is thought to be a good predictor of DBS outcome. Tykocki et al found a positive correlation coefficient of 0.40 between MLE due to MER and the results at six months with DBS on. ${ }^{6}$ Maltête et al showed that the MLE was an accurate independent predictor of six-month postoperative UPDRS III motor improvement in the off-medication/onstimulation condition after STN-DBS in PD. ${ }^{7}$ Mann et al addressed the effects of MER and DBS lead placement during DBS surgery in either the STN or the globus pallidus internus (GPi) and found these effects to be similar in magnitude to the effects observed four and six months after the surgery. ${ }^{16}$ Therefore, the positive correlation we found between the intraoperatively measured MLE and the DBS effect is concordant with the preceding literature. ${ }^{6,7}$

Our study yielded a Spearman's rho of 0.68 and 0.59 , while the data from Tykocki et al resulted in a rho of 0.40 . Differences in study design could explain differences in results. While Tykocki et al measured the MLE within 48 hours, in our study, we measured the MLE intraoperatively. During the days following electrode insertion edema develops. ${ }^{17}$ This might make clinical measurements less location specific and could explain a poorer correlation with the DBS effect during follow-up.

Furthermore, another difference in study design is that our study differentiates between the primarily and secondarily treated hemisphere, because of reported bilateral effects of electrode implantation. ${ }^{15,18,19}$ Since the insertion of two electrodes can be considered as two separate interventions, analyzing them separately may be a more accurate approach to analyze the MLE as a proxy for clinical effect. With this design, the MLE in the secondarily treated hemisphere could seem relatively small. Indeed, we found a tendency for the MLE in the primarily treated hemisphere to be larger than the MLE in the secondarily treated hemisphere. This finding is consistent with the theory that electrode implantation generates bilateral effects. It seems that the MLE comprises an overall effect, in which the MLE in the primarily treated hemisphere determines the effect direction.

\section{Limitations}

The MDS-UPDRS part III might have been preferable as the primary variable to assess the MLE rather than a modified (nonvalidated) version of the scale. However, due to the intraoperative setting, this was not possible. Nonetheless, items used in the modified scale represent core PD symptoms, making the modified scale a clinically relevant representation of disease severity. Since measurements were obtained during surgery, the influence of fatigue or stress could not be precluded, as well as the possible influence of anesthetic medication (eg, sedatives) administered to facilitate frame placement. Also, using two different methods for converting UPDRS 3.0 scores to MDS-UPDRS III scores (Goetz for item scores ${ }^{9}$ and Hentz for total scores ${ }^{13}$ ) could potentially introduce bias. However, excellent agreement was reported between both conversion methods. ${ }^{13}$

Furthermore, the primarily treated hemisphere differs among patients. The left hemisphere was treated first in all but one of our patients, even though the most affected hemispheres were evenly distributed between left and right. As described previously, this was 
Table 3. Clinical Results.

Sum scores median (range)

Primarily treated hemisphere

Preinsertion

Postinsertion

Microlesion effect

Off med/On stim

DBS effect

Secondarily treated hemisphere

$4(0-8)$

$1(0-5)$

$3(0-7)$

$1(0-5)$

3 ( -1 to 7$)$

$4(0-8)$

$2(0-6)$

$1(0-4)$
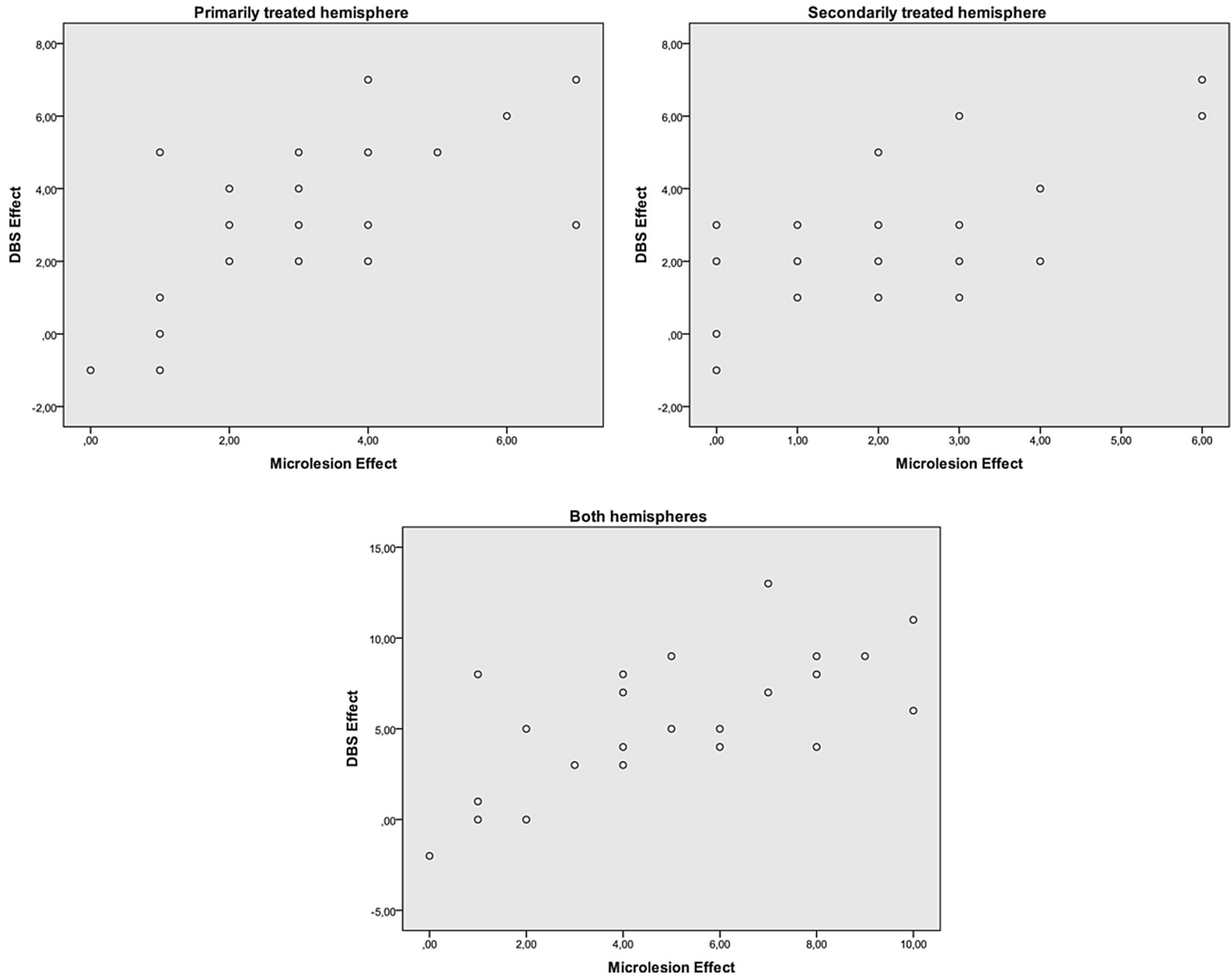

Figure 3. Correlation between the MLE and DBS effect for the primarily (left) and secondarily (right) treated hemisphere and for both hemispheres together (below).

done because of our standard practice. The finding that the primarily treated hemisphere displays the largest MLE advocates for treating the most impaired hemisphere first. This would allow optimal measurement of symptom reduction and therefore optimal placement of the electrode in the hemisphere that causes the worst symptoms.

Finally, follow-up duration differed among patients due to our clinical workflow. In our center, patients return to the outpatient clinic whenever they experience that their symptoms have returned to the presurgical state. Theoretically, in this fashion, the DBS effect would be measured without interference of a potentially still present MLE. Although no literature regarding the exact duration of the MLE exists, studies show that GPi-DBS in PD and dystonia patients can cause an MLE that lasts for $\sim 21$ days. ${ }^{1,8,20}$ However, it has been shown that STN targets show a greater MLE than GPi targets, ${ }^{16}$ enabling the possibility of STN-DBS causing a longer lasting MLE. It would therefore be possible that during the follow-up examination, some MLE would still be present. A partially present MLE could explain a higher correlation coefficient when comparing these results to the results of Tykocki et al. ${ }^{6}$ Ideally, all patients would have had a follow-up duration of $>6$ months, which is often considered to be an applicable follow-up duration for longterm DBS outcome. The correlation of the MLE and the long-term DBS effect was beyond the scope of this study, but will be 
investigated in the prospective longitudinal study that we are currently conducting.

\section{CONCLUSION}

In this study, we confirmed the presence of an intraoperatively quantifiable MLE during DBS implantation. This MLE has a clinically relevant correlation with the effect of DBS in PD patients. These results suggest that the MLE can be relied upon as evidence of a clinically effective DBS electrode placement.

\section{Acknowledgements}

The authors thank Ms. J. ten Kate for her help during data collection. The authors thank Dr. J. Vonk for her help with statistical methodology and analysis.

\section{Authorship Statements}

Stèfan F. Lange and Gea Drost designed the study. Stèfan F. Lange, Naomi I. Kremer, Gea Drost, Fiete Lange, and T. SteendamOldekamp are responsible for data collection. Stèfan F. Lange and Naomi I. Kremer are responsible for data analysis. Stèfan F. Lange and Naomi I. Kremer prepared the manuscript draft with important intellectual input from Teus van Laar, D.L. Marinus Oterdoom, Anthony R. Absalom, J. Marc C. van Dijk, and Gea Drost. All authors approved the final manuscript. Stèfan F. Lange, Naomi I. Kremer, and Gea Drost had complete access to the study data. All authors approved the final version of the manuscript.

\section{How to Cite This Article}

Lange S.F., Kremer N.I., van Laar T., Lange F., SteendamOldekamp T.E., Oterdoom D.L.M., Absalom A.R., van Dijk J.M.C., Drost G. 2023. The Intraoperative Microlesion Effect Positively Correlates With the Short-Term Clinical Effect of Deep Brain Stimulation in Parkinson's Disease. Neuromodulation 2023; 26: 459-465.
7. Maltête D, Derrey S, Chastan N, et al. Microsubthalamotomy: an immediate predictor of long-term subthalamic stimulation efficacy in Parkinson disease. Mov Disord. 2008;23:1047-1050.

8. Wang Y, Li P, Gong FL, Gao Y, Xu YY, Wang W. Micro lesion effect of the globus pallidus internus with deep brain stimulation in Parkinson's disease patients. Acta Neurochir. 2017;159:1727-1731.

9. Goetz CG, Tilley BC, Shaftman SR, et al. Movement Disorder Society-sponsored revision of the unified Parkinson's disease rating scale (MDS-UPDRS): scale presentation and clinimetric testing results. Mov Disord. 2008;23:2129-2170.

10. Kremer NI, Oterdoom DLM, van Laar PJ, et al. Accuracy of intraoperative computed tomography in deep brain stimulation-a prospective noninferiority study. Neu romodulation. 2019;22:472-477.

11. Schnider TW, Minto CF, Shafer SL, Gambus PL, Andresen C, Goodale DBYE. The influence of age on propofol pharmacodynamics. Anesthesiology. 1999:90:15021516.

12. Schnider TW, Minto CF, Gambus PL, Andresen C, Goodale DB, Shafer SLYE. The influence of method of administration and covariates on the pharmacokinetics of propofol in adult volunteers. Anesthesiology. 1998;88:1170-1182.

13. Hentz JG, Mehta SH, Shill HA, Driver-Dunckley E, Beach TG, Adler CH. Simplified conversion method for unified Parkinson's disease rating scale motor examinations. Mov Disord. 2015;30:1967-1970.

14. Chung SJ, Jeon SR, Kim SR, Sung YH, Lee MC. Bilateral effects of unilateral subthalamic nucleus deep brain stimulation in advanced Parkinson's disease. Eur Neurol. 2006:56:127-132

15. Tabbal SD, Mwiza Ushe BS, Minks JW, et al. Unilateral subthalamic nucleus stimulation has a measurable ipsilateral effect on rigidity and bradykinesia in Parkinson disease Exp Neurol. 2008:211:234-242.

16. Mann JM, Foote KD, Garvan CW, et al. Brain penetration effects of microelectrodes and DBS leads in STN or GPi. J Neurol Neurosurg Psychiatry. 2009;80:794-797.

17. Borellini L, Ardolino G, Carrabba G, et al. Peri-lead edema after deep brain stimulation surgery for Parkinson's disease: a prospective magnetic resonance imaging study. Eur J Neurol. 2019;26:533-539.

18. Alberts $\mathrm{J}$, Okun MS, Vitek JL. The persistent effects of unilateral pallidal and subthalamic deep brains on force control in advanced Parkinson's patients. Parkinsonism Relat Disord. 2009;14:481-488.

19. Agostino R, Dinapoli L, Modugno N, et al. Ipsilateral sequential arm movements after unilateral subthalamic deep-brain stimulation in patients with Parkinson's disease. Mov Disord. 2008;23:1718-1724.

20. Cersosimo MG, Raina GB, Piedimonte F, Antico J, Graff P, Micheli FE. Pallidal surgery for the treatment of primary generalized dystonia: long-term follow-up. Clin Neurol Neurosurg. 2008;110:145-150.

\section{COMMENTS}

The authors do a nice job in correlating an intraoperative microlesional effect with short-term outcomes in subthalamic nucleus (STN) deep brain stimulation (DBS) by limiting their analysis to patients undergoing electrode placement with a single pass with computed tomography $(\mathrm{CT})$ confirmation of appropriate localization. I look forward to seeing the long-term results in this patient cohort, which will be far more impactful.

Jason Schwalb, MD Township, MI, USA

\section{REFERENCES}

1. Cersosimo MG, Raina GB, Benarroch EE, Piedimonte F, Alemán GG, Micheli FE. Micro lesion effect of the globus pallidus internus and outcome with deep brain stimulation in patients with Parkinson's disease and dystonia. Mov Disord. 2009:24:1488-1493.

2. Holiga Š, Mueller K, Möller HE, et al. Resting-state functional magnetic resonance imaging of the subthalamic microlesion and stimulation effects in Parkinson's disease: indications of a principal role of the brainstem. Neurolmage Clin. 2015:9:264-274.

3. Jech R, Mueller K, Urgošík D, et al. The subthalamic microlesion story in Parkinson's disease: electrode insertion-related motor improvement with relative corticosubcortical hypoactivation in fMRI. PLoS One. 2012;7:e49056.

4. Singh A, Mehrkens $\mathrm{JH}$, Botzel K. Effect of micro lesions of the basal ganglia on ballistic movements in patients with deep brain stimulation. J Neurol Sci. 2012:314:175-177.

5. Chang SY, Shon YM, Agnesi F, Lee KH. Microthalamotomy effect during deep brain stimulation: potential involvement of adenosine and glutamate efflux. Proc 31st Annu Int Conf IEEE Eng Med Biol Soc Eng Futur Biomed EMBC 2009. 2009;3294-7.

6. Tykocki T, Nauman P, Koziara H, Mandat T. Microlesion effect as a predictor of the effectiveness of subthalamic deep brain stimulation for Parkinson's disease. Stereotact Funct Neurosurg. 2013;91:12-17.
This retrospective study further demonstrates the correlation of the micro-lesion effect (MLE) of insertion of DBS electrodes and the eventual palliative effects of DBS in the STN. In this case series, no microelectrode recording (MER) was carried out, which helps to dissociate the damaging effects of MER from the potentially less permanent lesional effects of insertion of the DBS electrode per se. The authors also excluded patients in who more than one attempt at DBS electrode insertion was carried out. The finding that one pass with a DBS electrode produced any MLE is somewhat surprising, but this may be due to the relative fragility of the STN given its small volume relative to the size of the electrode. Most of our DBS implants are in the globus pallidus (GP), placed under general anesthesia, and it is rare to see any clinically relevant MLE in outpatients post-operatively. The authors, findings that MLE correlated with the positive outcome of 
DBS is not surprising, since the basis of target selection, at least initially, pivoted on the known palliative effects of subthalamotomy, pallidotomy, and thalamotomy. It seems very clear now that DBS is much more than a lesional effect, producing bihemispheric improvement of motoric performance on a systemic basis. Whether the MLE is simply a coincident finding of target localization in STN, or representative of the mechanism of DBS in the STN, remains to be proven.

Kim Burchiel, MD Portland, OR, USA 